\title{
Assessing Material Fragility using Nanoscale Incremental Dynamic Analysis
}

\author{
Steve Cranford \\ Editor-in-chief, Matter \\ scranford@cell.com
}

\section{Abstract:}

Herein we present a paradigm shift in which material behavior can be assessed by performance, rather than intrinsic properties, based on a concept of material fragility. With concepts and design elements adapted from performance-based structural engineering, we propose the implementation of a computational nanoscale incremental dynamic analysis (NIDA) methodology to determine the failure risk of material systems at different loading thresholds, applied to a simple carbon nanotube case study. This is critical in emerging applications where load is highly unpredictable and nonuniform. Introducing a paradigm of performance-based materials design, systems can be interpreted beyond a static set of properties (e.g., ultimate strength, toughness) and optimized according to probable risk, loss of functionality, and/or complete failure, i.e. concepts of resilience and reliability. While a particular atomistic system is chosen as an example, in general, the proposed methodology can be applied to any stochastic load conditions and efficiently automated. 


\section{Introduction}

Materials are typically characterized by strength, when, in many applications, what we really want to know is how they will fail. Indeed, materials are often associated with a single exemplary property, and we hear proclamations such as: "Graphene, one of the strongest known materials, has an ultimate strength of 130 GPa." While the above statement is a demonstrated fact [1] (and an important characteristic of graphene), properties such as "strength" usually have an assortment of implicit constraints - i.e., graphene has a measured ultimate strength of $130 \mathrm{GPa}$... when tested via nanoindentation, with monotonically increasing indention depth at constant displacement rate, at a constant temperature... etc. Indeed, this is how materials are characterized by standard test methods.

Many real-life applications have more complicated loading cases. In practice, graphene in a device is unlikely to be indented at a constant rate just as a carbon nanotube is unlikely to be stretched in uniaxial tension. Particular loading cases and defined strengths may never be reached, nor even applicable. While important, predicting the strength of a material is not equivalent to predicting when or how a material system will fail. Many loading conditions are variable, stochastic, and combinatorial. There are known loads, alongside the unknown loads, alongside the "unknown unknown" loads. It is difficult to assign a single "magnitude" of load, yet we do it all the time in material and structural design (thus the need for conservative design factors). There exists a need for a fundamental change of nanomaterial characterization, from a strength-based assessment (i.e., the strength of material $X$ is $Y$ ) to a stochastic failure or performance-based assessment, asserting: the risk of failure of material $X$ subject to loading $Y$ at an intensity level $Z$ is $P \%$. This defines the material fragility.

As multiple disciplines use materials to drive innovation, load-bearing structural components (e.g., in a building or vehicle) have been typically grounded in proven systems. To achieve true progress, there must be a merging of designed material response with performance, e.g., new paradigms of design, development, and insertion of advanced engineering material systems, with intended downstream application [2,3]. A key recognition is that static values of strength is insufficient, as material assembly and configuration combined with combinatorial load conditions dictate ultimate capacity and failure modes [4]. As the performance objective of a structure increases (e.g., during hazards) the uncertainty increases to a point that requires a high level of conservatism in design $[5,6]$. At the same time, computational power has enabled incremental/iterative approaches - rather than a single data point, ranges of variables can be systematically explored to reduce uncertainty and conservatism through statistical analysis [7]. Introducing a paradigm of performance-based materials design, material systems can be interpreted beyond a static set of properties (e.g., ultimate strength, toughness) and designed according to (i) probable risk, (ii) loss of functionality, and/or (iii) complete failure.

A paradigm borrowed from macroscale structural performance-based design $[5,6,8,9]$, the concept enables the evaluation of complex materials systems, risk of failure, as well as tolerance and failure susceptibility. Such consideration is key in applications where loading is both highly unpredictable and nonuniform, including electromagnetic or radiation shielding, extreme temperature, high pressure, vibration or fatigue loading, and combinations thereof. Such loads are critical to emerging and transformative technologies in resource processing, clean energy generation and storage, aerospace, defense, medicine, and computation, where advanced materials have become essential [10]. This is in stark contrast to material components subject to anticipated uniform loads, such as uniaxial tension. Unpredictable loads result in unpredictable - and frequently premature - failure. 
How can one assess the reliability of a material system in such stochastic applications? Here we present the formulation and implementation of an innovative performance-based materials design methodology, encompassing: (1) development of a computational nanoscale incremental dynamic analysis (NIDA) protocol to determine the failure risk of material systems at different loading thresholds, as well as defining load-type tolerance factors, applied to; (2) the performance of a simple carbon nanotube subject to vibrational load as a proof-of-concept case study. Intended to be pedological, the key contribution of this work is the association and consideration of risk and fragility alongside materials failure.

\section{Methods}

Techniques from structural engineering are adopted to assess material systems. Embracing and extending concepts from performance-based structural engineering [5], NIDA will enable the comprehensive assessment of material behavior under stochastic loading conditions, allowing for the first time the evaluation of multiple limit states across a range of load levels/thresholds.

\section{Performance-Based Assessment}

At the structural scale, engineers have laid their trust on accepted building codes, practices, and standards [6]. This is currently not the case with material systems - there exists no standard or code for materials design. For buildings, recent disasters have indicated that significant damage can occur even when compliant with the building code - e.g., minimum design criteria may not ensure continued functionality. Likewise, so-called "strong" materials may not meet their ultimate limit states prior to loss of function. In order to meet needs, critical buildings should be designed according to criteria that results in continued and uninterrupted functionality (see Fig. 1). Such a performance-based paradigm can be applied to material systems. The term "performance" refers to a building's functional capacity after a disaster. Similarly, one can define the load threshold (or combinations thereof) applied to a material system with associated damage or loss of function.

It is noted that performance-based design was not intended as a universal substitute for design by traditional codes [11, 12]. Similarly, a performance-based materials approach is not proposed to replace traditional heuristics of strength or other material properties. Rather, it is an opportunity to enhance and tailor design to match objective applications, expectations, and quantify risk and reliability by accurately predicting materials performance under diverse load conditions. The process will consider the uncertainties inherent in quantifying the magnitude of potential loads, assessing the actual response of materials systems, and the potential effects of the performance on intended functionality. Specifically, a commonly used performance-based approach in structural engineering is adapted: incremental dynamic analysis, or IDA [5].

In non-linear dynamic analysis, the response of a structure is considered part of the time domain. Thus, ultimate strength and/or limit state can be considered not only a function of the maximum load/deformation, but also what happened before that state was reached. From a modeling perspective, the calculated response can be sensitive to the characteristics of the individual load record used as input. To mitigate sensitivity, several analyses are required using records to achieve a reliable estimation of the probabilistic distribution of system response. Moreover, as the properties of the response clearly depend on the intensity of the load, a comprehensive assessment calls for numerous analyses at various levels of intensity to represent different possible scenarios (e.g., increments). The necessity of such analysis is motivated by seismic events - e.g., there is no "ideal" earthquake. As a consequence, such a load cannot be treated as a typical engineering load for limit state design. Depending on the character of the earthquake (e.g., frequency, duration, etc.), a structure may collapse under a weaker magnitude than it 
was designed for. It was recognized that such a method can also enable checking for multiple limit-states, e.g. for life-safety, as well as levels of intensity that represent different threats, such as immediateoccupancy and collapse-prevention $[13,14]$.

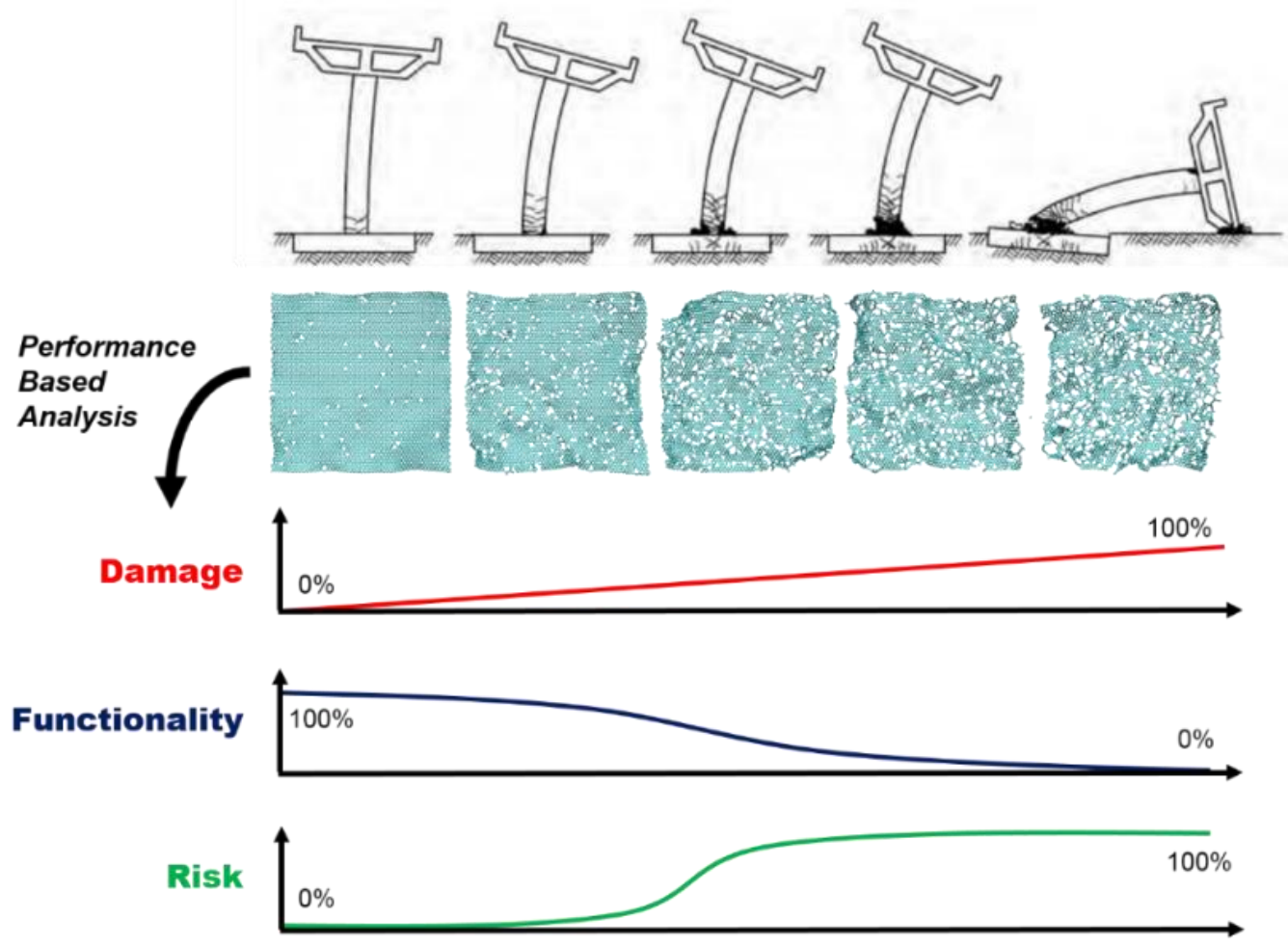

Figure 1: Performance-based assessment from structures (simple column) to materials (here, graphene as an example). While damage to a system can accumulate linearly (red), loss of functionality can be highly nonlinear (blue), resulting in and associated increased risk of failure (green).

In effect, IDA involves subjecting a system to a suite of load conditions, attempting to explore all possible load histories (or records), rather than a single ideal case or value. For Earthquake Engineering, IDA involves performing multiple nonlinear dynamic analyses of a structural model under a set of ground motions, each scaled to several levels of seismic intensity. For example, a weak seismic record recorded in Bolivia can be scaled and applied to a structure intended for Los Angeles. Appropriate post-processing can present the results in terms of IDA curves, one for each ground motion record, of the seismic intensity, typically represented by a scalar intensity measure (IM), versus the structural response, or damage measure (DM). The IM is smartly chosen well so that appropriate hazard maps (or curves) can be produced by probabilistic analysis. Common choices for seismic design include peak ground acceleration or peak ground velocity. The DM can be any structural response quantity that relates to structural or nonstructural damage, such as maximum inter-story drifts or peak floor accelerations. The intent here is to take the IDA framework and adopt it to general nanoscale material system design. 


\section{Nanoscale Incremental Dynamics}

Five key concepts from IDA are taken, and transformed from a structural to a material basis to implement as nanoscale incremental dynamics analysis, or NIDA:

1) Stochastic Load Records (LR) The key application for IDA is when single ideal load cases are insufficient to represent physical conditions - i.e., when the loading is highly uncertain, scattered, or probabilistic. For structural engineering, this clearly encompasses seismic loads, but also wind, flood, fire and other hazards. This requires a means to generate or collect a set of such load records, to apply to a system systematically. For materials, stochastic loadings can also be temporally (e.g., vibrations, heat flux) or spatially (e.g., impacts, radiation, local heat) varying. Representative records must be produced/sampled to assess system performance.

2) Intensity Measure (IM) Logically, a single load record results in a single assessment of system performance. To assess the effects across load records on system behavior, it is convenient to reduce a complex load history to a single defining parameter - i.e., an intensity measure (IM). The IM for earthquake records is commonly the spectral acceleration (or Richter scale). For vibration as an example, potential IMs include equivalent energy, frequency, displacement range, load duration, etc.

3) Load Scaling (Increment) Subjecting a system a single load record at a single intensity measure may or may not result in failure. As such, the record must be linearly scaled (by multiplying the IM by a factor) until system failure (or other performance threshold) is reached. This enables not only a relation between IM and failure state, but also how the system behaves under intermediate conditions, and enables probabilistic assessment at a given IM. For statistical relevancy, number of total records and IM increment must be carefully selected, balancing computationally efficiency and rigor.

4) Damage Measure (DM) The final critical parameter to assess the performance of a system is the damage measure - i.e., how one can assess not only failure but the accumulation of failure viz. damage. At the nanoscale, material damage measures can include maximum strain, fraction of broken bonds, loss of crystallinity/structure, etc. The key is to define a DM that can indicate not only when failure occurs, but when functionality deteriorates, enabling performance assessment at multiple thresholds.

5) Fragility Curves The result of multiple load records with incremental scaling(s) is a set of curves indicating failure (or critical damage) thresholds. Assuming sufficient data is produced across a spectrum of intensity measures, these curves can then be statistically analyzed to produce a fragility curve. The fragility curve can be considered the key result of the NIDA analysis - it represents the probability of failure given a loading scenario, regardless of the particular instantiation of the loading [15].

These concepts are incorporated within a simple loop-within-a-loop algorithm, where a set of load records (outer loop) is systematically applied to a system, and incrementally scaled (inner loop) until failure, recording performance for each load record (LR) at each intensity measure (IM). The NIDA process is summarized by Table 1 and Figure 2: 
Table 1: Summary of Proposed NIDA Procedure

\begin{tabular}{|c|c|c|}
\hline \# & Step & Description \\
\hline 1 & $\begin{array}{l}\text { System Design } \\
\text { (Input) }\end{array}$ & $\begin{array}{l}\text { Development of proposed material system archetype, including representative } \\
\text { system dimensions and boundary conditions (temperature, pre-stress or pre- } \\
\text { strain). Also includes modeling framework (e.g., here, full atomistic molecular } \\
\text { dynamics, encompassing definition of force fields and interaction potentials). } \\
\text { The system design stage incl. the definition of damage measure (DM) and } \\
\text { failure criteria. }\end{array}$ \\
\hline 2 & Generate & $\begin{array}{l}\text { Produce single instantiation of stochastic load record (LR, e.g., vibration and/or } \\
\text { radiation), including defining parameters and intensity measure (IM). }\end{array}$ \\
\hline 3 & Scale & Enter incremental scaling loop and scale LR by factor to achieve nominal IM. \\
\hline 4 & Simulate & $\begin{array}{l}\text { Subject system to nonlinear analysis. Requires computational model that can } \\
\text { accurately nonlinear response, limit states and progressive failure } \\
\text { mechanisms. In the current case, full atomistic molecular dynamics will be } \\
\text { applied. }\end{array}$ \\
\hline 5 & Assess & $\begin{array}{l}\text { Check damage state of system at end of LR; if damage exceeds critical state } \\
\text { (failure), exit scaling loop and generate new stochastic load. Repeat previous } \\
\text { process. If damage does not exceed critical state, scale load intensity to } \\
\text { increase IM, and repeat simulation. }\end{array}$ \\
\hline 6 & Iterate & Cycle through records until sufficient data is collected (for statistical analysis). \\
\hline 7 & $\begin{array}{l}\text { Summarize } \\
\text { (Output): }\end{array}$ & $\begin{array}{l}\text { Statistically evaluate system performance, damage states and intensity } \\
\text { measures to produce fragility curve reflecting probability of failure. }\end{array}$ \\
\hline
\end{tabular}

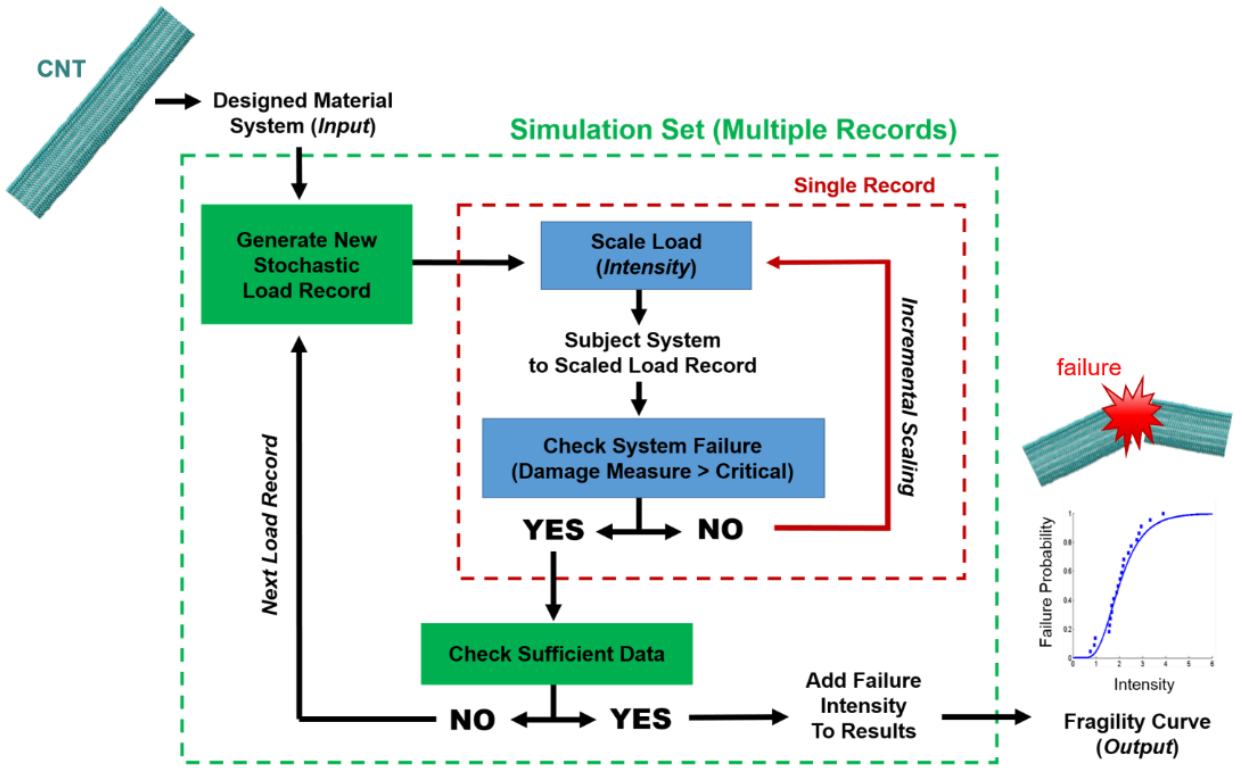

Figure 2: Schematic of NIDA procedure. Starting with a material system (here, a single CNT), an iterative loop-within-a-loop process is initiated. A stochastic load record is generated (at a low IM), and the system is modeled, incrementing the intensity until critical DM (e.g., failure) is observed (inner loop). The process repeats with new load records (outer loop), and data is compiled to produce a fragility curve. 


\section{Load-Type Tolerance Factors}

Treating failure as a random event requires that an engineer/designer must tolerate some finite risk of unacceptable performance, quantified by a system's probability of failure. The resulting fragility curve of the NIDA analysis is effectively a cumulative distribution function (CDF) indicating the probability of the material system will fail at a given random load with a prescribed intensity. To associate some definite metrics (and enable predictions of unsampled load intensities), an analytical expression for the CDF is required. "Choosing" a distribution can be somewhat of a qualitative and subjective process. The physics that underlie a problem should indicate an appropriate choice if possible (appealing to extreme value distributions to represent load and strength) [16-18].

Borrowing from material assessment, we propose to apply Weibull statistics to our method, widely implemented in the failure analysis of brittle materials, even at the atomistic scale [19-21]. The Weibull distribution is an extreme value distribution, governing the time to occurrence of the "weakest link" of many competing failure processes, and this makes the distribution an appropriate choice in representing the underlying probability distribution function for failure. It has been successful in applications such as ball bearings, relays, and general material fracture/fatigue. Here, we are looking to predict the minimum value of critical load intensity. The two-parameter Weibull CDF is given by the expression:

$F(\tau)=1-\exp \left[-\left(\frac{\tau}{\beta}\right)^{\eta}\right]$

for $\tau>0$. Related, the probability density function, or PDF, is given by:

$f(\tau)=\left(\frac{\eta}{\beta}\right)\left(\frac{\tau}{\beta}\right)^{\eta-1} \exp \left[-\left(\frac{\tau}{\beta}\right)^{\eta}\right]$

for $\tau>0$. Here $\eta$ (a scatter parameter) and $\beta$ (a central location parameter) are the two distribution parameters. Variation of these parameters result in a highly flexible distribution function (see Fig. 3a). The location or scale parameter, $\beta$, gives an indication of the range of critical intensities in which failure is observed and is related to the mean intensity of the PDF. In failure analysis, $\eta$ is typically denoted $m$ and known as the Weibull modulus. The modulus gives an indication of variance/uncertainty to failure. A high modulus indicates a high tolerance of a system to a given load type, whereas a low modulus indicates more uncertainty (Fig. 3b). Thus, in our formulation, we label the $\eta$-value as the load-type sensitivity or load-type tolerance of a given material system.

Note the fundamental difference for our application is that the variance in the traditional Weibull statistics is due to discrepancy in material samples (i.e., defect size, distribution, etc.) whereas the variance here is imposed on the uncertain load history to the same sample system. Thus, while the math is similar, the parameters do not reflect a variation in intrinsic material strength, but rather in sensitivity due to a particular load instantiation. It is key to note also that Weibull Analysis - and thus the derived parameters - is used on failures of the same mode. Critically, a Weibull plot only applies to one load variation. Thus, different $\eta$-values should be derived for different loads (e.g., vibration $v$. temperature, for example). There is a rich knowledge base of Weibull statistics to analyze material behaviors (including parameter estimation, estimate bias/invariance, determination of confidence bounds, etc.) which can be drawn from to develop the statistics of NIDA as applications expand. 

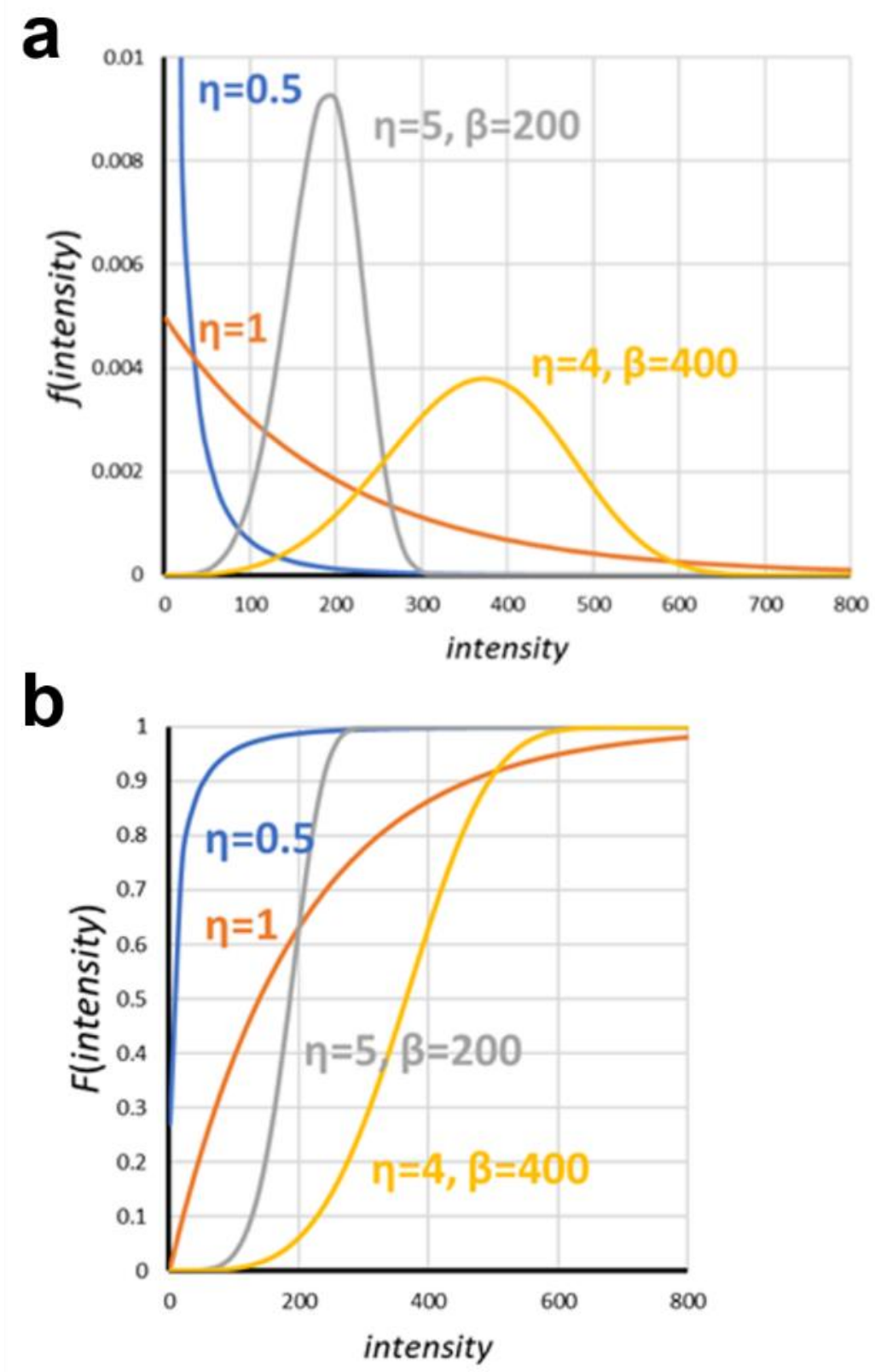

Figure 3: Weibull distribution functions for material fragility. (a) probability density function (PDF) from Eq. (2) with example $\boldsymbol{\eta}$ (a scatter parameter) and $\boldsymbol{\beta}$ (a central location parameter) values; (b) cumulative distribution function (CDF) from Eq. (1) with same values, illustrating range of potential system behaviors to represent.

\section{Case Study}

To illustrate the proposed NIDA methodology, as proof-of-concept, we present an easily modeled nanoscale structure: a single $(8,8)$ carbon nanotube (CNT), approx. $10 \mathrm{~nm}$ in length (Fig. 4), subject to axial vibration. The CNT could reflect a component of a nanodevice [22], such as a sensor, subject to extreme environmental conditions, for example. The relative well-known behavior of carbon nanotubes applied here simply provided a stable material platform/system to demonstrate the NIDA approach. 


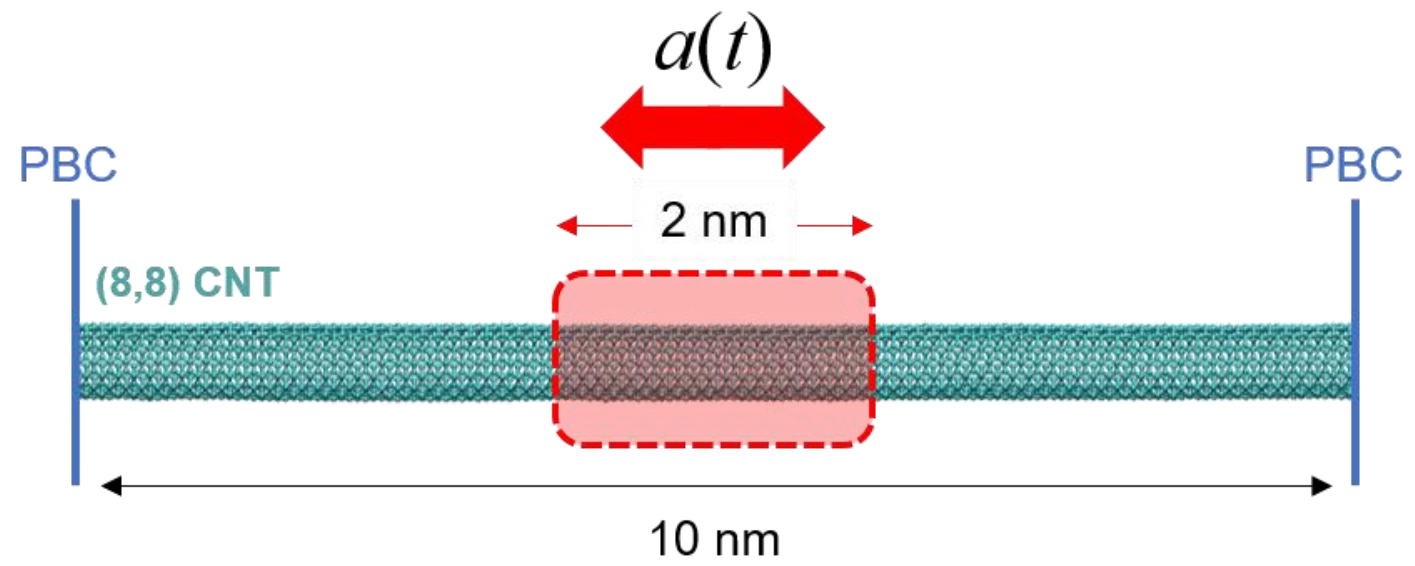

Figure 4: Full atomistic model of $(8,8)$ carbon nanotube with load and boundary conditions.

Why vibration? Vibration can be thought of as a mechanical proxy to reflect the non-local effects of various loads such as radiation. Say, for example, a system is subject to radiation exposure over a large area, the scale of MD limits the size of the system that can be successfully modeled. As a radiation field is not likely limited to an area on the order of square-nanometers, we can also apply the indirect loading of the system, caused by irradiation of surrounding bulk, which results in KE waves and thermal fluctuations, manifesting as mechanical vibration/excitations. Similar to seismic loading, we can subject the system to vibrations by imposing an additional acceleration on each particle in the system (e.g., global acceleration), or, alternatively, at specified boundaries. Computationally, this load is typically considered a "gravity" term acting on the atoms/particles, and scales with the mass of the atom(s). By Newton's law, it follows that: $F_{\text {vibration }}=m a(t)$ where $a(t)$ is a randomly constructed acceleration response (e.g., load records). Benefits of constructing acceleration responses as a stochastic load include the ability to construct vibrations in three orthogonal components (xyz), and define characteristics such as peak acceleration, duration, and frequency content of the load record. To enable scaling of the vibrational load, we consider the power of the excitation $(P)$, converting to equivalent applied energy as the IM, where:

$P=\frac{d}{d t} K E=m v(t) a(t) \rightarrow K E=m v(t) a(t) d t$

where $m$ is the atomic mass, $v$ the velocity magnitude, and $a$ the prescribed acceleration. Noting the relationship between kinetic energy (KE) and temperature, we can solve for effective atomic velocity, $v_{e}$, at a known temperature, or:

$\frac{1}{2} m v_{e}^{2}=\frac{3}{2} k_{B} T \rightarrow v_{e}=\sqrt{\frac{3 k_{B} T}{m}}$

where $k_{\mathrm{B}}$ is the Boltzmann constant and $T$ is the temperature. Substituting the above relation, the energy of the applied vibrational field can be determined via the instantaneous temperature of the system, the applied acceleration, and the time increment, where: $U_{\text {vibration }}=\left(\sqrt{3 k_{B} T m}\right) a(t) d t$. The vibrational 
energy is a function of the temperature, which can be fixed (or varied) as a system variable. For our case here, a fixed temperature is used, and thus the IM reduces to the applied atomistic acceleration, $a(\mathrm{t})$.

Full atomistic molecular dynamics simulations were implemented via open-source software LAMMPS [23]; carbon-carbon interactions were modeled via the ReaxFF potential ( $a b$ initio based) [24]; the $(8,8)$ CNT consisted approximately 2500 atoms; a timestep of 0.05 femtoseconds was used, with full periodic boundaries (CNT continuous/infinite along axis) using an NVT ensemble @ 300K; the system was minimized upon simulation initiation and equilibrated at $300 \mathrm{~K}$ for 100,000 timesteps; stress was calculated via virial stress in the axial direction $[25,26]$; each LR was run for six scale increments at 250,000 steps each $(1,500,000$ steps per LR), using 8 processors per run, taking approximately 2 days per load record.

\section{$\underline{\text { Results and Discussion }}$}

The simulation approach can be summarized as follows: (1) a load record (LR) is generated, (2) the CNT is subjected to the LR scaled to the minimum level $(0.25 \mathrm{~g}),(3)$ if failure is recorded, the simulation is halted, if no failure is recorded, the LR is scaled by $+0.25 \mathrm{~g}$, (4) the simulation is repeated, system assessed for failure, steps (3) and (4) repeated until failure observed, (5) record failure intensity, (6) move to subsequent LR.

\section{Load Generation and Scaling}

As a stochastic load, we choose vibration/accelerations in the axial direction, reducing the problem to 1D conditions. We generate random vibrational time histories (load records, or LRs), effectively subjecting the CNT to a "nano-earthquake". The record was generated using a uniform random distribution from $1.00 \mathrm{~g}$ to $+1.00 \mathrm{~g}$, selecting 20 values for each record, and scaled accordingly. Similar to earthquakes, the intensity measure (IM) is chosen as peak acceleration, using increments of $+0.25 \mathrm{~g}$, for applied random acceleration magnitudes of $\pm 0.25 \mathrm{~g}$ to $\pm 1.50 \mathrm{~g}$ (resulting in a maximum 6 iterations per LR). A sample randomly generated load record is presented in Figure 5. The effective frequency of the record is determined by the duration of applied accelerations (here, approximately $1 \mathrm{THz}$ ).

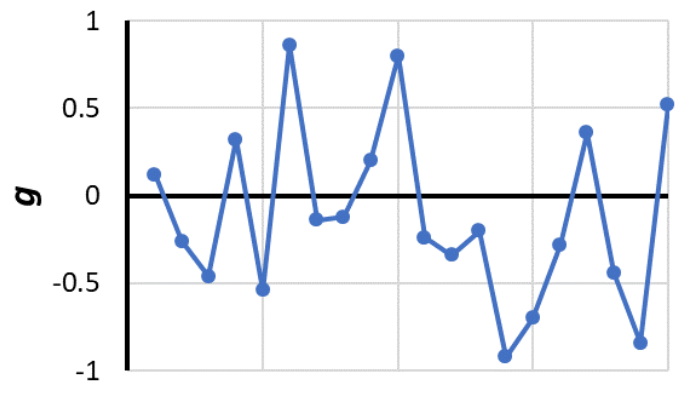

Figure 5: Random generated acceleration load record (LR) for vibration study. $X$-axis (timescale) is arbitrary, and scaled according to the simulation. Here the accelerations were applied at a frequency of approximately 1 $\mathrm{THz}$ (i.e., time between plotted points equal to $10^{-12}$ seconds).

\section{Onset of Failure}

Next, we need a suitable damage measure (DM) to define failure. Damage/failure was determined visually (e.g., molecular trajectory snapshots) as well as by tracking both axial stress and potential energy over each LR at each intensity. From stress, failure could be determined when an increased intensity resulted in a decrease in stress magnitude (indicating the system could no longer carry stress due to significant 
cracks). From potential energy ( $P E)$, a damaged system did not return to its ground state after the onset of damage (e.g., broken bonds were present). Tracking the change in energy, $\Delta E$, indicated accumulation of damage, as well as a setting a critical threshold to define complete failure (Figure 6).

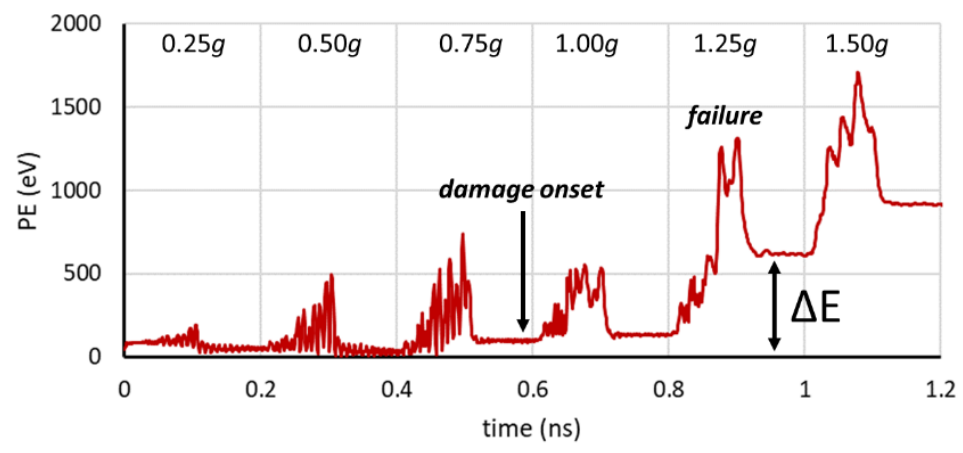

Figure 6: Potential energy (PE) over each load intensity (single LR); onset of damage \& failure defined by $\Delta E$.

\section{Fragility}

While only four LRs were generated, failure occurred at three distinct intensity levels (one at $0.75 \mathrm{~g}$, one at $1.00 \mathrm{~g}$, and two at $1.25 \mathrm{~g}$ ). This resulted in sufficient data to plot a preliminary fragility curve and undertake Weibull analysis (Figure 7). This resulted in a vibrational load sensitivity factor of $\boldsymbol{\eta}=\mathbf{5 . 5 8}$ and a vibration scale parameter of $\boldsymbol{\beta}=\mathbf{1 . 0 3}$ for the $(8,8)$ nanotube. Utilizing Eq. (1):

$$
F(\tau)=1-\exp \left[-\left(\frac{\tau}{1.03}\right)^{5.58}\right]
$$

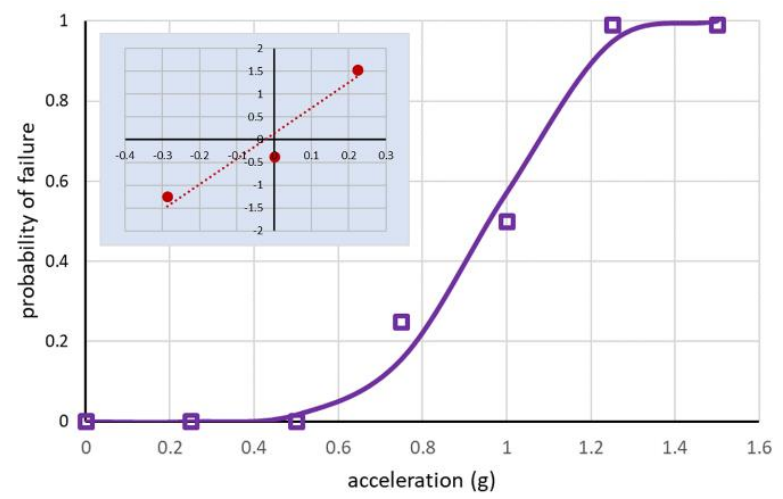

Figure 7: Fragility curve and fitted CDF. Inset: Log-log plot for parameter fitting.

This fitted analytical form now enables prediction, such as: the risk of failure of an $(8,8)$ carbon nanotube subject to random accelerations with a maximum intensity of $1.1 \mathrm{~g}$ at $1 \mathrm{THz}$ is $76.4 \%$. Note that $1.10 \mathrm{~g}$ was never simulated/modeled. 


\section{Sensitivity}

How sensitive are these parameters to initial boundary conditions? As a trial, we subjected the CNT to an initial pre-strain of $1 \%$ and repeated the NIDA process. One could predict that the addition of a prestrain/pre-stress would weaken the system, as well as decrease the range of critical intensities. Indeed, both effects are reflected in the results, with a reduced sensitivity factor of $\eta=5.43$ (a $3 \%$ reduction) and a vibration scale parameter of $\beta=0.94$ (a $9 \%$ reduction) when subject to $1 \%$ pre-strain (see Figure 8 ). Now we can conclude: the risk of failure of an $(8,8)$ CNT with $1 \%$ pre-strain subject to random accelerations with a max. intensity of $1.1 \mathrm{~g}$ at $1 \mathrm{THz}$ is $90.4 \%$.We see that the $1 \%$ pre-strain has increased the risk of failure by $14 \%$.

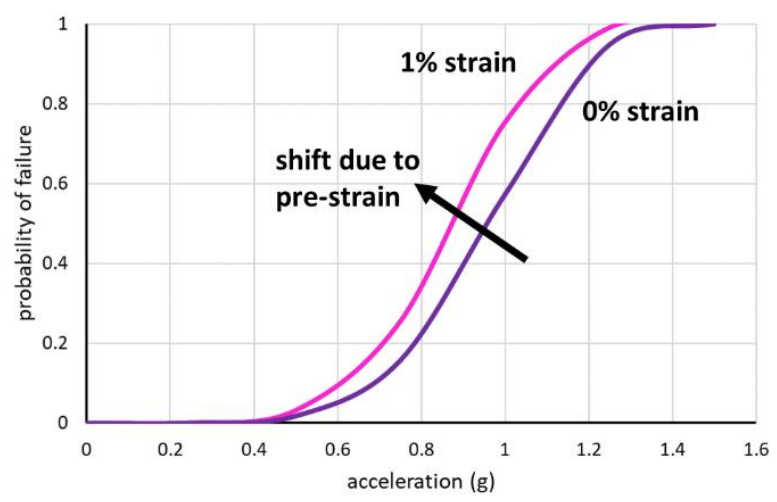

Figure 8: Fragility curve and fitted CDFs of CNT with $0 \%$ and $1 \%$ pre-strain.

It is clear that four load records (with only 20 data points) and a relatively large increment of $0.25 \mathrm{~g}$ is much too coarse of a framework to make any conclusive statistical analysis or conclusions for the carbon nanotube. However, significant computational effort was required - four records $\times$ six intensities each = twenty-four total full atomistic MD simulations for these preliminary results (repeated in totality for the effect of pre-strain) - suggesting the necessary effort for larger, more complex systems. Indeed, this small study is only intended as a proof-of-concept, to illustrate the NIDA methodology. For full characterization, an application with association boundary conditions and predicted load ranges is necessary, encompassing more load records with more data points, applied with finer increments, to construct a more rigorous fragility curve. To extend the current work here is moot without a particular case or application. Simple variations - such as a range of pre-strain values and size effects $(e . g .$, a $(10,10)$ CNT) - could also be easily considered to map the change of $\eta$ and $\beta$ versus strain.

In addition to radiation and vibration loads, both temperature and static load cases could easily be considered in combination with generated stochastic records. Temperature could be controlled via classical thermostatting approaches. The thermostatting can be used to either suppress temperature fluctuations (e.g., constant temperature) or systematically increase/decrease average temperature (e.g., representing a nuclear reactor overheating, for example). Static loading (e.g., a constant force and/or applied deformation) can be used to represent system pre-stresses and mechanical loads prior to stochastic effects. Similar to classical boundary value problems, such loads can be applied by fixed boundaries, prescribed stresses, or affine strain fields. A complete assessment of the materials performance requires systematic combinations of each load contribution (e.g., radiation + vibrations + temperature + pre-stress) to mimic the physical conditions the system would be subject to in situ, and delineated any coupling effects that may arise. For example, there is a clear coupling between the temperature and applied vibration, due to the magnitude of KE of the atoms pre-excitation. The influence 
on ultimate strength or deformation behavior is difficult to determine a priori, without rigorous simulation and statistical analysis provided by the proposed NIDA procedure.

\section{Conclusion}

The methodology here enables a stochastic approach to assess the performance of the material. Rather than a single data point of strength, the NIDA approach will produce fragility curves that reflect anticipated damage as a function of load threshold (via scaled combinatorial loadings). The aim is to link effective performance to relevant statistical loading fields that demonstrate a scientific path and design methodology to optimize materials with specific risk thresholds.

The described NIDA protocols can be applied to various material structures, including single crystals, heterogeneous structures, interface of phases and grain boundaries, encompassing ceramics, metals, and/or hybrid systems. Moreover, the proposed methodology can be applied to any stochastic load conditions. A major challenge for materials experts is to develop predictive capabilities that provide the designer with information regarding the damage extent and consequences across the system service lifetime. Here, the resulting fragility curves can be used to describe system behavior and enable statistical assessment of risk and reliability for a given system. The general goal is to apply a systematic methodology to predict and optimize the behavior of material systems driven by probabilistic performance metrics rather than static material properties. A fragility-based assessment provides more specific information for a particular case but is less general. It is intended not to be an intrinsic material property, but rather a materials design tool once a particular use-case (and thus load-case) is determined. Indeed, this approach does not replace material properties such as strength (necessary to define and understand materials), but rather supplement the assessment of material response in a particular system subject to a particular loading scenario, associating the risk (and thus design confidence) to a particular load intensity or combination [6].

In addition to specific use cases, fragility curves are useful to compare performance metrics across both materials and load type. For example, one could ask are carbon nanotubes less susceptible than copper nanowires to vibrational damage? Radiation damage? By how much? To quantify the sensitivity of materials and material systems to variations in load (either by type or combination), we've introduced a flexible statistical framework to define and quantify load-type tolerance factors (similar, in practice, to the concept of flaw-tolerance). Ultimately, the procedure is to be automated, requiring definition of input (model system, load records) and analysis parameters (damage measure, failure criteria, load scaling increments), resulting in output (fragility curve), exploiting simulation parallelization.

While the focus is limited to nanoscale responses here (via MD), the basic approach is not limited to scale (indeed, borrowed from macroscale structural engineering), and can be formulated for nanoscale or microscale systems. This method can successfully bring performance-based structural engineering concepts to the material scale, to shift the paradigm of materials-by-design. As stated earlier, static material properties from idealized tests are limited in use for the design of complex material systems. Critical is how the system actually performs when subject to extreme loads and damage - often due to highly complex and variable events. The need for measurements of transient phenomena are essential to understanding mesoscale phenomena and the trend is [27]: "...that measurements that are dynamic, in situ, \& multi-modal will replace the static single-property characterization now common in macro \& nano regimes..." A key result is that the material performance - e.g., failure thresholds and limit states becomes a variable in the design process, enabling more robust/resilient systems, and exploiting predictive behaviors and failure modes, recognizing the intimate link between material behavior and response beyond the long-held paradigm of "stronger is better" and subsequently consider the material fragility. 


\section{Acknowledgements}

It is noted this concept was previously submitted as a grant proposal. S.W.C. felt the idea was worthwhile to publish for the community, will not be working on the methodology in the future, and invites other researchers to extend the work/application. S.W.C. acknowledges prior support from Northeastern University. Also, his wife for putting up with him as he ran simulations from home. Current employer, Cell Press, had no influence on this study. The calculations and the analysis were carried out using a Dell multi-core laptop workstation.

\section{References}

1. Lee, C., X. Wei, J.W. Kysar, and J. Hone, Measurement of the Elastic Properties and Intrinsic Strength of Monolayer Graphene. Science, 2008. 321(5887): p. 385-388.

2. S. Cranford, M.J. Buehler, Biomateriomics, 2012 (Springer, New York)

3. SW Cranford, J De Boer, C Van Blitterswijk, MJ Buehler, Materiomics: An -omics Approach to Biomaterials Research. Advanced Materials. 2013, 25 (6): p. 802-824

4. Cranford, S.W. and M.J. Buehler, Shaky foundations of hierarchical biological materials. Nano Today, 2011. 6(4): p. 332-338.

5. Vamvatsikos, D., Cornell, A., Incremental Dynamic Analysis. Earthquake Engineering and Structural Dynamics, 2002, Volume 31, Issue 3, Pages 491-514

6. Abbie B.Liel, Curt B. Haselton, Gregory G. Deierlein, Jack W. Baker, Incorporating modeling uncertainties in the assessment of seismic collapse risk of buildings. Structural Safety, 2009, Volume 31, Issue 2, March 2009, Pages 197-211

7. YC Hsu, $\mathrm{CH} \mathrm{Yu,} \mathrm{MJ} \mathrm{Buehler,} \mathrm{Using} \mathrm{deep} \mathrm{learning} \mathrm{to} \mathrm{predict} \mathrm{fracture} \mathrm{patterns} \mathrm{in} \mathrm{crystalline} \mathrm{solids.}$ Matter, 2020, 3 (1), p. 197-211

8. Vamvatsikos, D., Fragiadakis, M., Incremental dynamic analysis for estimating seismic performance sensitivity and uncertainty. Earthquake Engineering and Structural Dynamics, 2010, 39(2), pp. 141163

9. Kazantzi, A.K., Vamvatsikos, D., Lignos, D.G., Seismic performance of a steel moment-resisting frame subject to strength and ductility uncertainty. Engineering Structures, 2014, Volume 78, Pages 69-77

10. Materials Genome Initiative for Global Competitiveness. 2011; Available from: https://www.whitehouse.gov/mgi.

11. Bozorgnia, Y. and V.V. Bertero, Earthquake engineering : from engineering seismology to performance-based engineering2004, Boca Raton, Fla.: CRC Press.

12. Krawinkler, H., Deierlein, G.G., Challenges Towards Achieving Earthquake Resilience Through Performance-Based Earthquake Engineering. Geotechnical, Geological and Earthquake Engineering, 2014, 32, pp. 3-23

13. Cook, D.T., Liel, A.B., DeBock, D.J., Haselton, C.B., Benchmarking FEMA P-58 repair costs and unsafe placards for the Northridge Earthquake: Implications for performance-based earthquake engineering. International Journal of Disaster Risk Reduction, 2021, 56, 102117 
14. Liel, A.B., Deierlein, G.G., Cost-benefit evaluation of seismic risk mitigation alternatives for older concrete frame buildings. Earthquake Spectra, 2013, 29(4), pp. 1391-1411

15. Porter, K., R. Kennedy, and R. Bachman, Creating fragility functions for performance-based earthquake engineering. Earthquake Spectra, 2007. 23(2): p. 471-489.

16. C. Lu, R. Danzer, F.D. Fischer. Fracture statistics of brittle materials: Weibull or normal distribution. Phys. Rev. E, 65 (6) (2002), Article 067102

17. A.D.S. Jayatilaka, K. Trustrum. Statistical approach to brittle fracture. J. Mater. Sci., 12 (7) (1977), pp. $1426-1430$

18. Danzer, R., Supancic, P., Pascual, J., Lube, T. Fracture statistics of ceramics-Weibull statistics and deviations from Weibull statistics. Eng. Fract. Mech., 74 (18) (2007), pp. 2919-2932

19. Roman, R.E., Cranford, S.W. Defect sensitivity and Weibull strength analysis of monolayer silicene. Mechanics of Materials, 2019, Volume 133, Pages 13-25

20. N.M. Pugno, R.S. Ruoff. Nanoscale Weibull statistics. J. Appl. Phys., 9 (2) (2006), Article 024301

21. N.M. Pugno, On the strength of the carbon nanotube-based space elevator cable: from nanomechanics to megamechanics. J. Phys. Condens. Matter, 18 (33) (2006), p. S1971

22. Baughman, R.H., A.A. Zakhidov, and W.A. de Heer, Carbon Nanotubes--the Route Toward Applications. Science, 2002. 297(5582): p. 787-792.

23. Plimpton, S., Fast Parallel Algorithms for Short-Range Molecular-Dynamics. Journal of Computational Physics, 1995. 117(1): p. 1-19.

24. Chenoweth, K., A.C.T. van Duin, and W.A. Goddard, ReaxFF reactive force field for molecular dynamics simulations of hydrocarbon oxidation. Journal of Physical Chemistry A, 2008. 112(5): $p$. 1040-1053.

25. D.H. Tsai. Virial theorem and stress calculation in molecular-dynamics. J. Chem. Phys., 70 (3) (1979), pp. 1375-1382.

26. Zimmerman, J.A., Webb III, E.B., Hoyt, J.J., Jones, R.E., Klein, P.A., Bammann, D.J.. Calculation of stress in atomistic simulation. Model. Simul. Mater. Sci. Eng., 12 (4) (2004), pp. S319-S332

27. Hemminger, J., G. Crabtree, and J. Sarrao, A Report from the Basic Energy Sciences Advisory Committee. U.S. Department of Energy, 2012: p. 1601-1606. 